\title{
Factors affecting fouling of an inorganic membrane during sweet whey ultrafiltration
}

\author{
Christine TADDEI *, P. AIMAR **, G. DAUFIN *, V. SANCHEZ ** \\ * INRA, Laboratoire de Recherches de Technologie laitière \\ 65, rue de Saint-Brieuc, 35042 Rennes Cedex, France \\ ** CNRS, Laboratoire de Chimie Physique et d'Electrochimie \\ 118, route de Narbonne, 31062 Toulouse Cedex, France
}

\section{Summary}

The effect of sweet whey concentration and composition on fouling was studied during ultrafiltration at $50^{\circ} \mathrm{C}$ through an inorganic membrane (Carbosep M4 type). The experimental variations in the course of time of flux, protein rejection and membrane resistance were analysed for several solutions and operating conditions. It is likely that protein adsorption and convective deposition of particles of solid matter are involved in membrane fouling by whey. Both phenomena occured at the very beginning of the ultrafiltration run. Their respective extent and kinetics depended on whey composition and concentration as well as on operating conditions.

The effect of several whey components on membrane fouling was also shown : soluble proteins, suspended particules, residual fat, calcium salts precipitating during ultrafiltration. Moreover, protein rejection was related to fouling type and operating conditions.

The results obtained with concentrated wheys should lead to an improvement of operating conditions in a three-stage ultrafiltration plant. A compromise solution between high fluxes and high protein rejection must be choosen to limit protein losses since the membrane is only partly retentive for whey proteins.

Key words : Fouling - Ultrafiltration - Whey.

\section{Résumé}

\section{Facteurs influençant l'encrassement d'une membrane minérale lors de l'ultrafiltration de lactosérum doux}

L'objectif de ce travail est d'étudier l'influence de la concentration et de la composition d'un lactosérum doux sur le transfert de matière lors de l'ultrafiltration à $50^{\circ} \mathrm{C}$ sur membrane minérale (Carbosep, Type M4). Le transfert de matière est caractérisé par des mesures de flux, rétention en protéines ( $\beta$-lactoglobuline, $\alpha$-lactalbumine), résistance hydraulique de la membrane. Cette dernière est un témoin de l'encrassement irréversible de la membrane. La composition du lactosérum est modifiée par centrifugation, clarification et complexation du calcium. Une solution de $\beta$-lactoglo- 
buline est également utilisée. L'analyse des variations des grandeurs représentatives du transfert en fonction du temps pour plusieurs lactosérums (concentrés et prétraités) et différents couples $(\mathrm{U}, \Delta \mathrm{P})$ précise la nature des phénomènes limitants impliqués dans le colmatage : adsorption de protéines et dépôt de matière par convection.

Ces phénomènes interviennent dès le début de l'ultrafiltration. Leur ampleur et cinétique respectives dépendent de la concentration et de la composition du lactosérum et également des conditions opératoires. Plusieurs constituants du lactosérum participent au colmatage : protéines solubles, particules en suspension, matière grasse résiduelle et sels de calcium qui peuvent précipiter en cours d'ultrafiltration.

A partir des résultats obtenus sur les lactosérums concentrés, quelques tendances se dégagent pour la conduite d'une installation d'ultrafiltration. Les conditions opératoires $(\mathrm{U}, \Delta \mathrm{P})$ doivent être modulées suivant l'étage de concentration considéré pour limiter les pertes en protéines dans le cas de membranes partiellement rétentives. II apparaît également nécessaire de définir des compromis flux-rétention.

Mots clés: Encrassement - Ultrafiltration - Lactosérum.

\section{Introduction}

Ultrafiltration is a pressure-driven membrane separation process. Since it does not require any change of state or high temperature it is particularly interesting for concentrating and/or separating biomolecules the diameter of which lies within the range 2 and $300 \mathrm{~nm}$.

One of the numerous applications of ultrafiltration is linked to whey protein concentration (DE BoER and HIDDINK, 1980 ; ZALL, 1982). Whey is the supernatant liquid produced in cheese-making or in casein production. It contains about $6 \mathrm{~g} \cdot \mathrm{l}^{-1}$ proteins particularly valuable for their functional and nutritional properties (Forsum, 1977 ; Melachouris, 1984).

Generally two kinds of problems must be faced in ultrafiltration : decline in flux in the course of time and partial solute rejection. Most studies on whey ultrafiltration concern flux improvement by fluid pretreatment (MULLER and Hayes, 1973 ; Hayes et al, 1974 ; Lee and Merson, $1976 \mathrm{a}, \mathrm{b}$; Fauquant et al., 1985) or testing membranes of different materials and configurations (Hiddink and van der WaAl, 1984 ; Cheryan and Kuo, 1984). A critical review of these investigations has been published (TADDEI, 1986). It shows that flux depends on concentration polarization together with membrane fouling and is affected by whey composition. shifting of physico-chemical equilibria, operating conditions and membrane material or geometry. Several explanations have been suggested for membrane fouling by whey. Some authors proposed the formation of a deposit on the membrane surface (LEE and Merson, 1976b ; Hayes et al., 1974 ; Kuo and CHERYAN, 1983), with a possible fouling within the membrane pores (MaUboIs, 1980; Kuo and CHERYAN, 1983). Others considered the formation of a gel-layer as the main «foulant » (HIDDINK et al., 1981; Howell et al., 1981). Furthermore, Howell et al. (1981) have suggested that protein adsorption could act as a gel growing factor.

Some models have been proposed to describe the flux limitation for ultrafiltration of protein solutions (BLATT et al., 1970; KOZINSKY and LightFoot, 1972 ; Matthiasson, 1983 ; Baklouti et al., 1984) but none of 
them was able to predict variations. Accordingly, a better knowledge of mass transfer during whey ultrafiltration is required to design and to control an industrial plant.

We recently studied the effect of operating parameters (flow velocity and applied pressure) and time on fouling during sweet whey ultrafiltration through an inorganic membrane (TADDEI et al., 1986). The experimental variations in the course of time of permeation fluxes, rejection of soluble proteins and membrane fouling may be explained by three phenomena : building-up of a polarization layer, membrane fouling by an adsorption phenomenon which involves physico-chemical interactions between solutes such as proteins and membrane and physical deposition of insoluble particles the size of which is greater than that of pores.

In this work, we emphasize the effect of whey protein concentration and modifications of protein environment on fouling, using the already described method. The various fluids used allowed to verify our previous assumptions concerning the limiting phenomena (TADDEI et al., 1986). Our results could be used to improve productivity of ultrafiltration plants.

\section{Experimental}

The experimental part has already been described (TADDEI et al., 1986 ; TADDEI, 1986).

The membrane was a Carbosep M4 type kindly supplied by SFEC (Société Française d'Eléments Catalytiques - Bollène, France). It was a composite membrane made of $\mathrm{ZrO}_{2}$ deposited inside a porous carbone tube (int. diameter : $6 \mathrm{~mm}$; length : $120 \mathrm{~cm}$ ) and with a cut-off of 20000 Dalton.

\section{A. Solutions}

Sweet, concentrated and pretreated wheys were used. Their composition is reported in table 1 (analysis methods appear in TADDEI et al., 1986).

Two wheys concentrates (retentates) were obtained by sweet whey ultrafiltration at $50{ }^{\circ} \mathrm{C}$ on a Romicon hollow-fiber module (PM 50 membranes $-5 \mathrm{~m}^{2}$ - $\left.2 \cdot 10^{5} \mathrm{~Pa}\right)$. The first retentate $\left(\mathrm{R}_{3}\right)$ corresponded to a concentration factor $\mathrm{CF}=3(\mathrm{CF}=$ initial volume $/($ initial volume - volume of permeate removed $))$; for the second one, $\mathrm{CF}=8\left(\mathrm{R}_{8}\right)$.

Clarified whey was prepared by thermocalcic aggregation of residual fat (FAUQUANT et al., 1985).

These fluids were stored at $-20^{\circ} \mathrm{C}$.

For some experiments, whey and $R_{3}$ retentate were centrifuged (30 $000 \mathrm{~g}$ $\left.20{ }^{\circ} \mathrm{C}-30 \mathrm{~min}\right)$.

In order to complex calcium, sodium citrate was added to clarified whey with a ratio of 2 citrate moles per calcium mole. 
TABLE 1

Chemical composition $\left(g \cdot l^{-1}\right)$ and physico-chemical characteristics of wheys and UF retentates

Composition chimique $\left(g \cdot l^{-1}\right)$ et caractéristiques physicochimiques des lactosérums et des rétentats d'ultrafiltration

\begin{tabular}{|c|c|c|c|c|}
\hline & $\begin{array}{l}\text { Sweet } \\
\text { whey }\end{array}$ & $\begin{array}{c}\text { Clarified } \\
\text { whey }\end{array}$ & $\underset{\text { Retentate }}{\mathrm{R}_{3}}$ & $\begin{array}{c}\mathbf{R}_{8} \\
\text { Retentate }\end{array}$ \\
\hline Total dry matter & 63.2 & 59.6 & 76.0 & 120.0 \\
\hline Ash & 5.1 & 6.8 & 4.95 & 11.0 \\
\hline Total nitrogen $\times 6.38$ & 8.1 & 6.6 & 21.4 & 56.0 \\
\hline$\beta$-lactoglobulin & 3.84 & 3.6 & 11.3 & 30.3 \\
\hline$\alpha$-lactalbumin & 0.87 & 0.8 & 2.4 & 4.8 \\
\hline $\mathbf{P}$ & 0.4 & 0.32 & 0.39 & - \\
\hline $\mathrm{pH}$ & 6.3 & 5.95 & 6.2 & 6.2 \\
\hline (Before centrifugation $(*)$ & 0.32 & 1.03 & 0.36 & - \\
\hline \multirow{3}{*}{$\mathrm{Ca}\left\{\begin{array}{l}\text { Supernatant } \\
\text { Difference }\end{array}\right.$} & 0.31 & 0.99 & 0.35 & - \\
\hline & 0.01 & 0.04 & 0.01 & - \\
\hline & \multicolumn{4}{|c|}{ Turbidity ${ }^{(1)}$} \\
\hline Before centrifugation & 0.50 & 0.01 & 5.50 & - \\
\hline After centrifugation & 0.32 & 0.01 & 0.20 & - \\
\hline Suspended matter ${ }^{(* *)}$ & & & & \\
\hline$\left(\right.$ dry matter $\left.\mathrm{g} \cdot \mathrm{kg}^{-1}\right)$ & 3.8 & 3.8 & 5.9 & - \\
\hline
\end{tabular}

(*) Centrifugation $30,000 \mathrm{~g}, 30 \mathrm{~min} ; 20^{\circ} \mathrm{C}$.

(**) Centrifugation $50,000 \mathrm{~g} ; 30 \mathrm{~min} ; 20^{\circ} \mathrm{C}$

(1) Absorbance at $600 \mathrm{~nm}$.

We also prepared a $\beta$-lactoglobulin solution at $3.0 \mathrm{~g} \cdot \mathrm{1}^{-1}$. The pure protein, obtained according to the method of PIERRE and FAUQUANT (1986), was dissolved in a synthetic ultrafiltrate ensuring a $0.1 \mathrm{M} \cdot \mathrm{kg}^{-1}$ ionic strength $\left(\mathrm{g} \cdot \mathrm{1}^{-1}\right)$ :

- lactose : 50.00
- urea : 1.00

- $\mathrm{Na}_{3}$ citrate : 2.50

$-\mathrm{NaCl}: 4.85$

$\mathrm{pH}$-value was adjusted to 6.2 with $3 \mathrm{~N} \mathrm{HCl}$.

In some cases, suspended particles were characterized by turbidity (Absorbance at $600 \mathrm{~nm}$ ) and analytical centrifugation $\left(50000 \mathrm{~g}-20^{\circ} \mathrm{C}-30 \mathrm{~min}\right)$ : weight of residue and calcium analysis of supernatant (table 1). The supernatant was diphasic except for clarified whey.

All the solutions were ultrafiltered in the presence of $1 \% \mathrm{NaN}_{3}$ to prevent bacterial growth. 


\section{B. Operating conditions}

The values for operating parameters were chosen close to those encountered in industrial conditions :

- tangential flow velocity, $\mathrm{U}\left(\mathrm{m} \cdot \mathrm{s}^{-1}\right): 1.8 ; 3.0 ; 4.0 ; 6.0$

- transmembrane pressure, $\Delta \mathrm{P}\left(10^{5} \mathrm{~Pa}\right): 1.0 ; 2.0 ; 4.0 ; 6.0$

- temperature, $\left({ }^{\circ} \mathrm{C}\right): 50$

- time, $\mathrm{t}(\mathrm{min}): 2$ to 240

\section{Procedure}

All the experiments were performed with the same membrane. Flux and rejection of soluble proteins ( $\beta$-lactoglobulin $\mathrm{MW}=36000 ; \alpha$-lactalbumin $\mathrm{MW}=16000)$ were measured versus time for various combinations $(\mathrm{U}, \Delta \mathrm{P})$. At the beginning and at the end of each experiment, pure-water flux $\mathbf{J}_{\mathrm{w}}$ was measured after 5 min tap water prerinsing $\left(50{ }^{\circ} \mathrm{C}\right.$; flow velocity and transmembrane pressure values equal to those used during ultrafiltration run) followed by $15 \mathrm{~min}$ water rinsing $\left(50{ }^{\circ} \mathrm{C} ; 1.8 \mathrm{~m} \cdot \mathrm{s}^{-1} ; 2 \cdot 10^{5} \mathrm{~Pa}\right)$. The hydraulic resistance $R_{h}$ was deduced from Darcy's law :

$$
\mathrm{J}_{\mathrm{w}}=\frac{\Delta \mathrm{P}}{\mu_{\mathrm{w}} \cdot \mathrm{R}_{\mathrm{h}}}
$$

$\mu_{w}=$ water dynamic viscosity $=0.54 \cdot 10^{-3} \mathrm{~Pa} \cdot \mathrm{s}$ at $50{ }^{\circ} \mathrm{C}$.

The initial value, $R_{h, j}$, of the membrane was $7.8 \cdot 10^{12} \mathrm{~m}^{-1}$. The increased resistance, $\Delta \mathrm{R}_{\mathrm{h}}$, consecutive to an ultrafiltration run indicated an irreversible fouling of the membrane, which is susceptible to be removed only after chemical or physical cleaning

$$
\Delta \mathrm{R}_{\mathrm{h}}=\mathrm{R}_{\mathrm{h}, \mathrm{f}}-\mathrm{R}_{\mathrm{h}, \mathrm{i}}
$$

$\mathrm{R}_{\mathrm{h}, \mathrm{f}}$ is the final hydraulic resistance.

\section{Membrane cleaning procedure}

Membrane cleaning was performed using an acid solution $\left(\mathrm{HNO}_{3} 0.06 \mathrm{M}\right.$; $50{ }^{\circ} \mathrm{C} ; 2 \mathrm{~m} \cdot \mathrm{s}^{-1} ; 2.0 \cdot 10^{5} \mathrm{~Pa}$ ) followed by $0.35 \mathrm{M} \mathrm{NaOH}$ with sodium hypochlorite at $0.16 \mathrm{~g} \cdot \mathrm{1}^{-1}$ of $\mathrm{Cl}_{2}\left(50^{\circ} \mathrm{C} ; 2 \mathrm{~m} \cdot \mathrm{s}^{-1} ; 2.0 \cdot 10^{5} \mathrm{~Pa}\right)$. This membrane cleaning procedure allows to recover the initial hydraulic resistance of the membrane.

\section{Results}

\section{A. Influence of whey concentration on mass transfer}

As illustrated in figures 1 and 2 , all the curves $J=f(t)$ exhibit the same shape whatever the protein concentration and operating conditions $(\mathrm{U}, \Delta \mathrm{P})$. When increasing CF from 1 to 8 , flux decreased. Moreover, $\mathrm{J}$ increased with increasing values of $\Delta \mathrm{P}$ or $\mathrm{U}$ (fig. 1 and 2). 


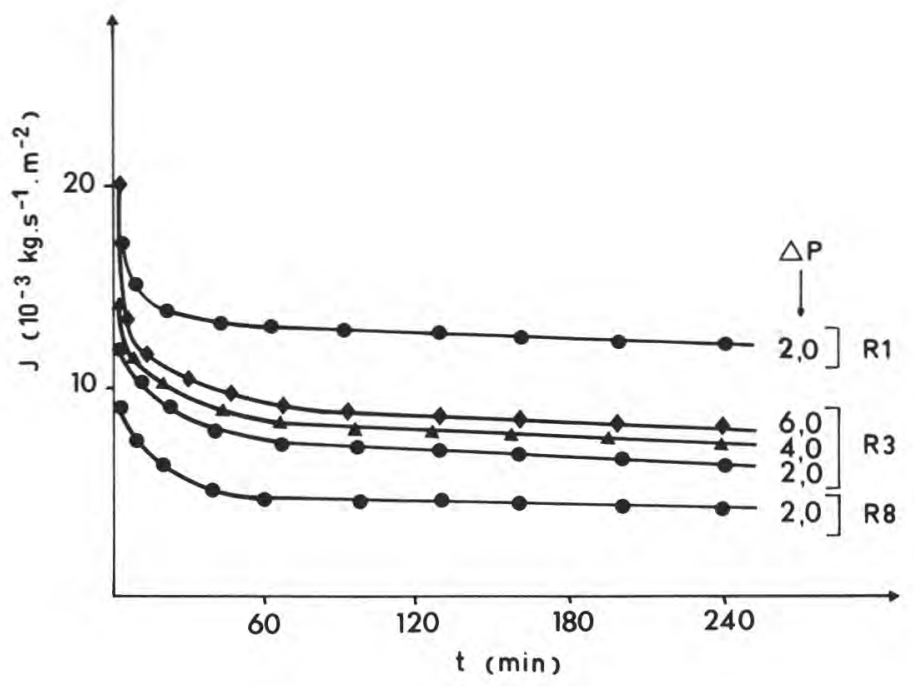

FIG. 1

Flux $(\mathrm{J})$ versus time under various pressures $\Delta P\left(10^{5} \mathrm{~Pa}\right)$ during ultrafiltration of initial $\left(R_{1}\right)$ sweet whey, $R_{3}$ and $R_{8}$ retentates through $M_{4}$ Carbosep membrane, $\left(\theta=50^{\circ} \mathrm{C}\right.$, flow velocity $=1.8 \mathrm{~m} \cdot \mathrm{s}^{-1}$ )

Densité de flux en fonction du temps à différentes pressions $\Delta P\left(10^{5} \mathrm{~Pa}\right)$ pendant l'ultrafiltration de lactosérum doux $\left(R_{1}\right)$ et de rétentats $R_{3}$ et $R_{8}$ sur membrane Carbosep $M_{4}\left(\theta=50^{\circ} \mathrm{C}\right.$, vitesse tangentielle $\left.=1,8 \mathrm{~m} \cdot \mathrm{s}^{-1}\right)$.

$$
\left(10^{-3} \mathrm{~kg} \cdot \mathrm{s}^{-1} \cdot \mathrm{m}^{-2} \# 3.6 \mathrm{l} \cdot \mathrm{h}^{-1} \cdot \mathrm{m}^{-2}\right) \text {. }
$$

Whatever the protein concentration, rejection of both proteins generally decreased during the first hour, then decreased or increased slightly or remained constant (fig. 3). For higher $\Delta \mathrm{P}$ (fig. 3a) or lower $\mathrm{U}$ (fig. 3b) values, protein rejection was more important.

For given operating conditions $(\mathrm{U}, \Delta \mathrm{P})$, the value of protein rejection was not generally much concentration dependent although a slight decrease in rejection might sometimes appear, especially for $\alpha$-lactalbumin in $R_{3}$ and $R_{8}$ retentates (fig. 3c). Variations in protein rejection were thus related to the membrane cut-off (20000 Daltons here) but also to the limiting phenomena observed.

The rapid increase of the hydraulic resistance $R_{h}$ within the first hour lowers down afterwards (fig. 4). The hydraulic resistance increased with increasing protein concentration. For instance, $R_{h . f}$ was seven times greater than $R_{h . i}$ for $R_{8}$ retentate when ultrafiltration was performed at the lowest flow velocity $\left(1.8 \mathrm{~m} \cdot \mathrm{s}^{-1}\right)$ and the highest pressure $\left(6.010^{5} \mathrm{~Pa}\right)$. Whatever the protein concentration, higher $\Delta \mathrm{P}$ or lower $\mathrm{U}$ levels resulted in higher $\mathrm{R}_{\mathrm{h} . \mathrm{f}}$ values. There- 


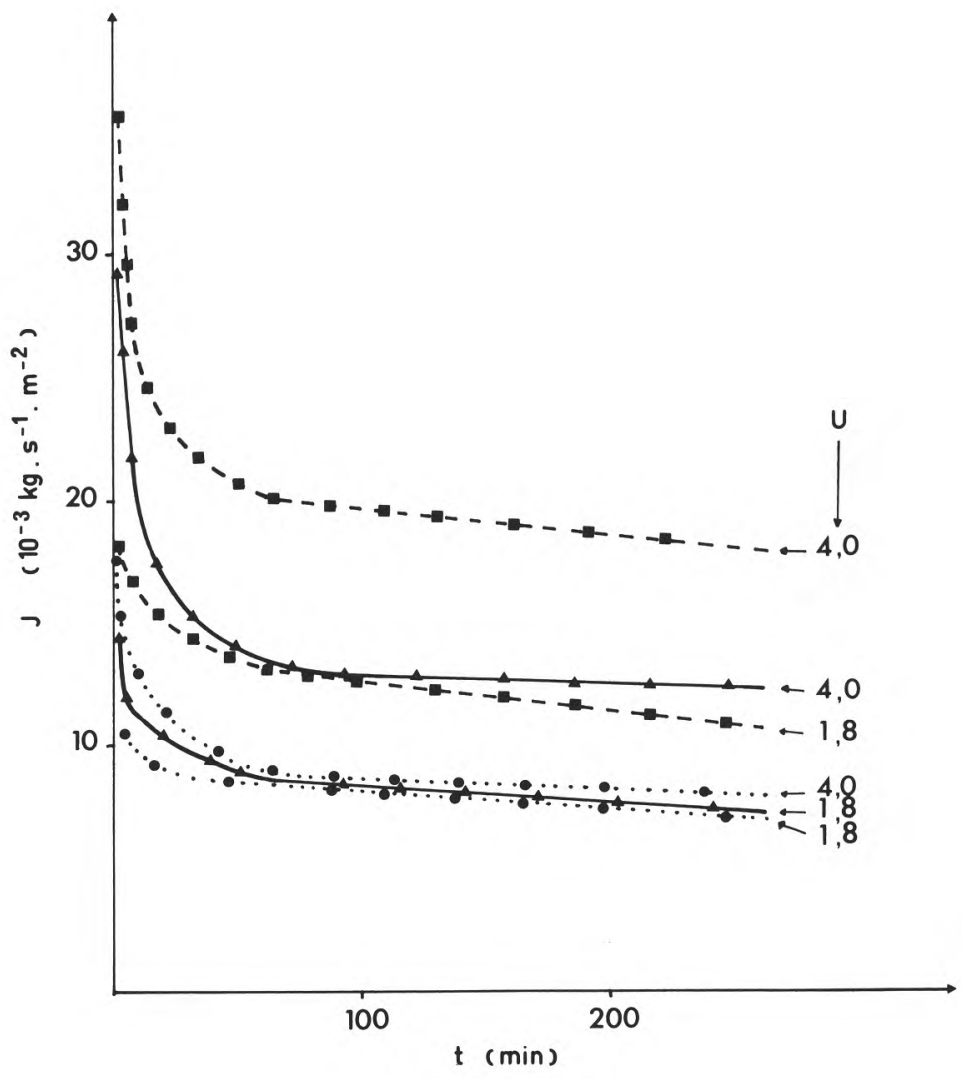

FIG. 2

Flux $(J)$ versus time with various tangential flow velocities $U\left(m \cdot s^{-1}\right)$ during ultrafiltration through $\mathrm{M}_{4}$ Carbosep membrane $\left(\theta=50^{\circ} \mathrm{C}\right.$, transmembrane pressure $\left.=4.0 \cdot 10^{5} \mathrm{~Pa}\right)$.

Sweet whey

$R_{3}$ Retentate

$R_{8}$ Retentate

$\Delta$
$\Delta \quad$ Rétentat $R_{3}$
Rétentat $R_{8}$

Densité de flux $(J)$ en fonction du temps à différentes vitesses tangentielles $U\left(m \cdot s^{-1}\right)$ lors de l'ultrafiltration sur membrane Carbosep $M_{4}$

$\left(\theta=50^{\circ} \mathrm{C} ;\right.$ pression transmembranaire $\left.=4,0 \cdot 10^{5} \mathrm{~Pa}\right)$.

$$
\left(10^{-3} \mathrm{~kg} \cdot \mathrm{s}^{-1} \cdot \mathrm{m}^{-2} \# 3.6 \mathrm{l} \cdot \mathrm{h}^{-1} \cdot \mathrm{m}^{-2}\right) .
$$


a)

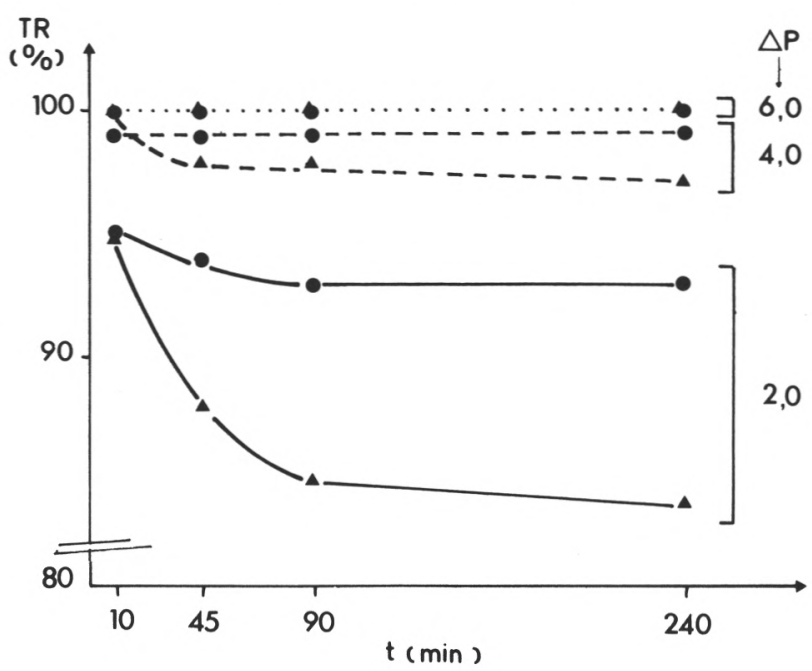

b)

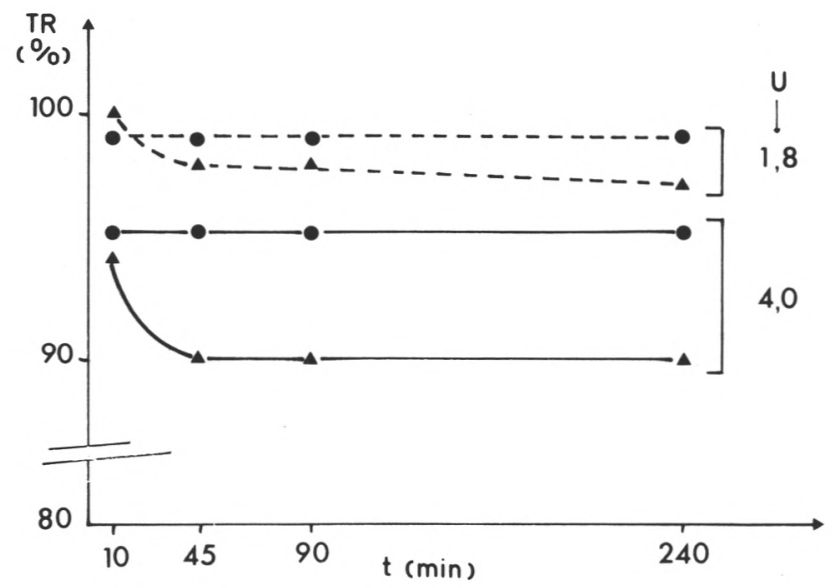

c)

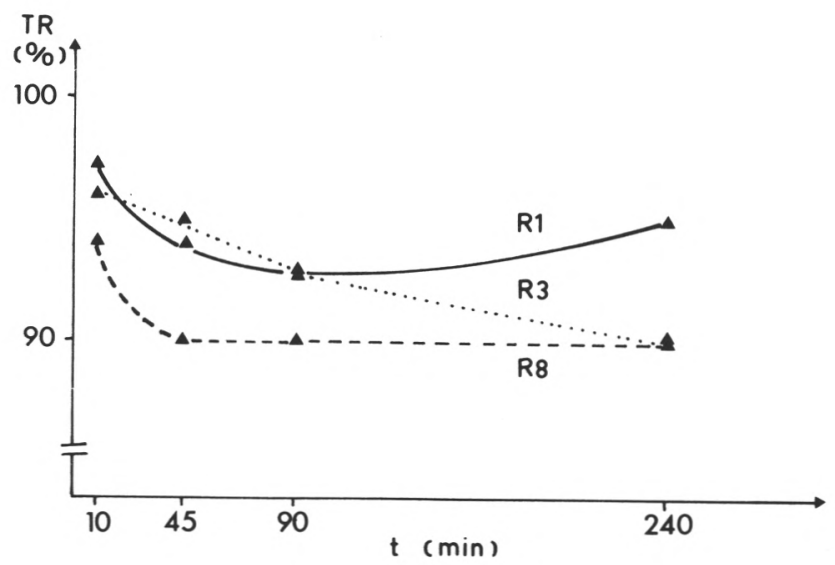




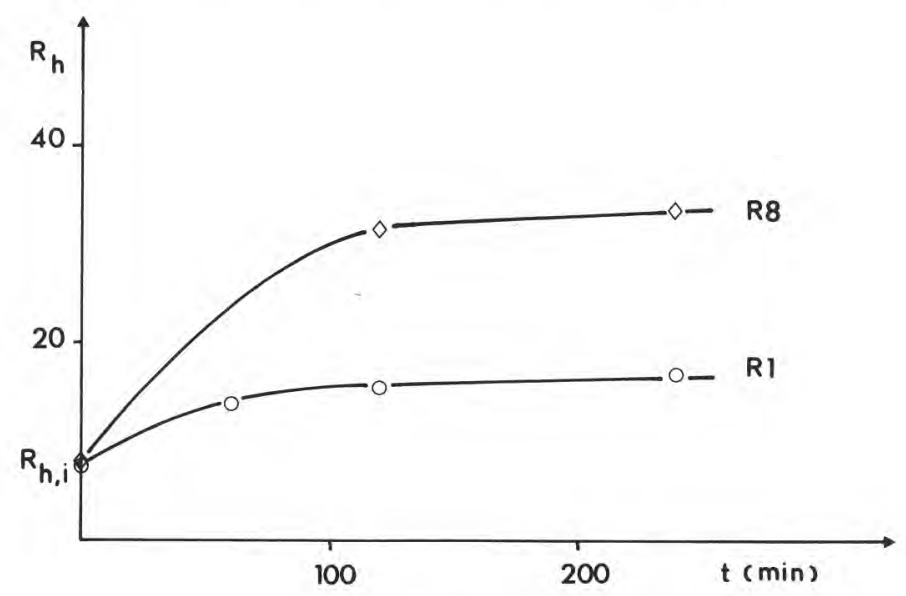

FIG. 4

Variation in final hydraulic resistance $R_{h . f}\left(10^{12} \mathrm{~m}^{-1}\right)$ measured after various ultrafiltration times $t$ (min) at $50^{\circ} \mathrm{C}$ on $M_{4}$ Carbosep membrane:

(O) : $R$, sweet whey: $U=3.0 \mathrm{~m} \cdot \mathrm{s}^{-1}-\Delta P=2.0 \cdot 10^{5} \mathrm{~Pa}$

$(\diamond): R_{8}$ retentate : $U=4.0 \mathrm{~m} \cdot \mathrm{s}^{-1}-\Delta P=2.0 \cdot 10^{5} \mathrm{~Pa}$

Initial hydraulic resistance

Résistance hydraulique initiale $\quad R_{h . i}=7.8 \cdot 10^{12} \mathrm{~m}^{-1}$

Evolution de la résistance hydraulique finale $R_{h . f}\left(10^{12} \mathrm{~m}^{-1}\right)$ mesurée après différents temps $t$ (min) d'ultrafiltration à $50{ }^{\circ} \mathrm{C}$ sur membrane Carbosep $M_{4}$ :

(O) : Lactosérum $R_{I}: U=3,0 \mathrm{~m} \cdot \mathrm{s}^{-1}-\Delta P=2,0 \cdot 10^{5} \mathrm{~Pa}$

$(\diamond)$ : Rétentat $R_{8}: U=4,0 \mathrm{~m} \cdot \mathrm{s}^{-1}-\Delta P=2,0 \cdot 10^{5} \mathrm{~Pa}$

FIG. 3

Rejection (TR) of $\beta$-lactoglobulin (-) and $\alpha$-lactalbumin ( $\mathbf{\Delta})$ versus time during ultrafiltration at $50{ }^{\circ} \mathrm{C}$ through $M_{4}$ Carbosep membrane of:

$3 a-R_{8}$ retentate under various pressures $\Delta P\left(10^{5} \mathrm{~Pa}\right)$; tangential flow veloci$t y=1.8 \mathrm{~m} \cdot \mathrm{s}^{-1}$

$3 b-R_{8}$ retentate with various flow velocity $U\left(m \cdot s^{-1}\right)$ (transmembrane pressu$r e=4.0 \cdot 10^{5} \mathrm{~Pa}$ )

$3 c$ - Sweet whey, $R_{1}, R_{3}$ and $R_{8}$ retentates ; tangential flow velocity $=4.0 \mathrm{~m} \cdot \mathrm{s}^{-1}$; pressu$r e=4.0 \cdot 10^{5} \mathrm{~Pa}$.

Taux de rétention (TR) de la $\beta$-lactoglobuline (-) et de l' $\alpha$-lactalbumine ( $\mathbf{\Delta}$ ) en fonction du temps lors de l'ultrafiltration à $50{ }^{\circ} \mathrm{C}$ sur membrane Carbosep $M_{4}$ de :

$3 a$ - Rétentat $R_{8}$ à différentes pressions $\Delta P\left(10^{5} \mathrm{~Pa}\right)$; (vitesse tangentielle $=1,8 \mathrm{~m} \cdot{ }^{-1}$ )

$3 b$ - Rétentat $R_{8}$ à différentes vitesses tangentielles $U\left(\mathrm{~m} \cdot \mathrm{s}^{-1}\right)$ (pression $\left.=4,0 \cdot 10^{5} \mathrm{~Pa}\right)$

$3 c$ - Lactosérum $R_{I}$ et rétentats $\left(R_{3}, R_{8}\right)$ (vitesse tangentielle $=4,0 \mathrm{~m} \cdot \mathrm{s}^{-1}$; pression $\left.4,0 \cdot 10^{5} \mathrm{~Pa}\right)$. 

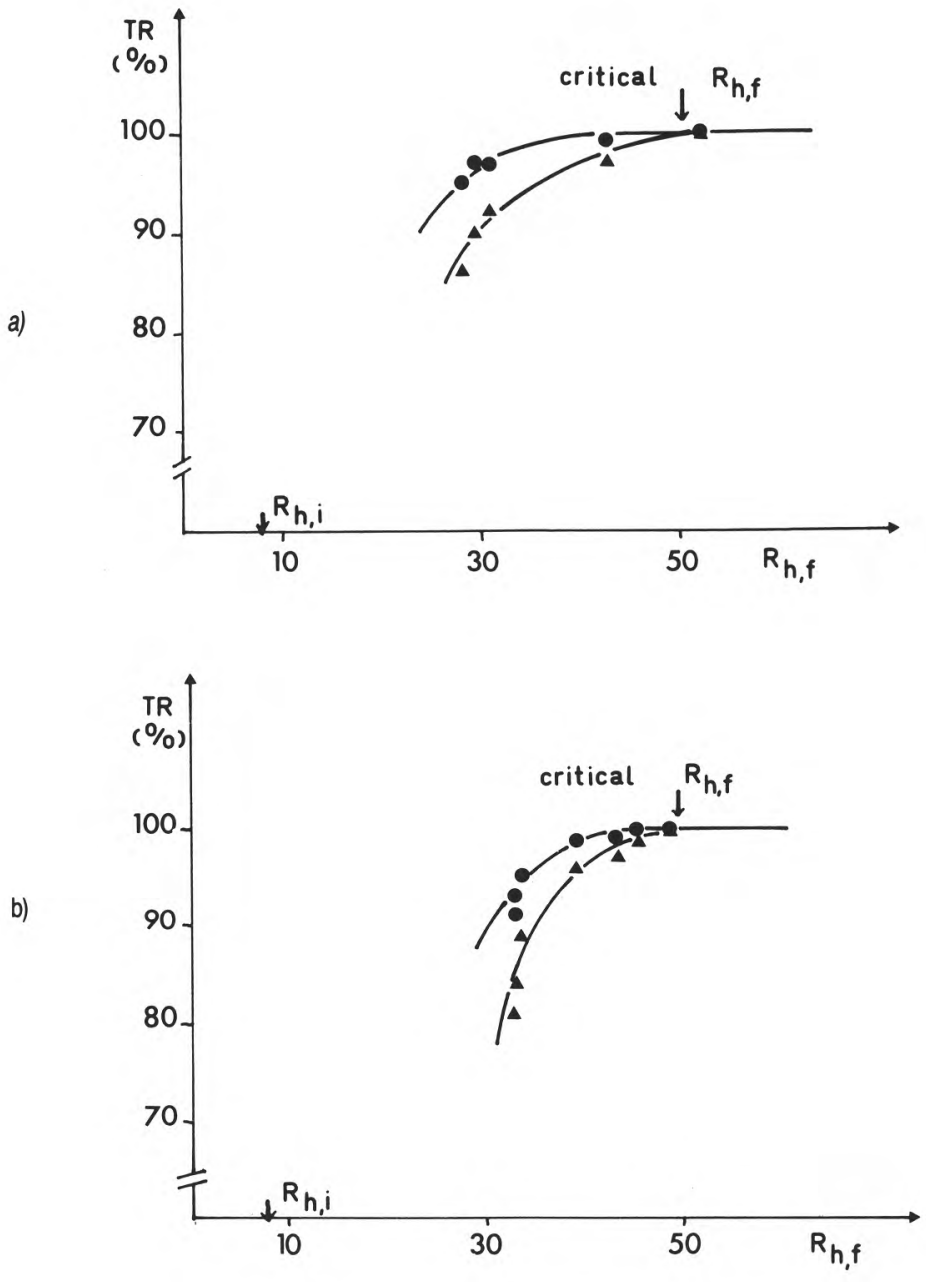

FIG. 5

Rejection (TR) of $\beta$-lactoglobulin (-) and $\alpha$-lactalbumin ( $\mathbf{\Delta})$ versus final hydraulic resistance $\left(R_{h . f}\right)$, after a 4 hour ultrafiltration at $50^{\circ} \mathrm{C}$ on $M_{4}$ Carbosep membrane.

$5 a-R_{3}$ retentate

$5 b-R_{8}$ retentate

(initial hydraulic resistance of clean membrane $R_{h . i}=7.8 \cdot 10^{12} \mathrm{~m}^{-1}$ ).

Taux de rétention (TR) de la $\beta$-lactoglobuline (O) et de l' $\alpha$-lactalbumine ( $\mathbf{\Delta})$ en fonction de la résistance hydraulique finale $\left(R_{h, f}\right)$ après 4 heures d'ultrafiltration à $50^{\circ} \mathrm{C}$ sur $5 a-$ Rétentat $R_{3}$ $5 b$ - Rétentat $R_{8}$ (résistance hydraulique initiale de la membrane propre $R_{h . i}=7,8 \cdot 10^{12} \mathrm{~m}^{-1}$ ). 


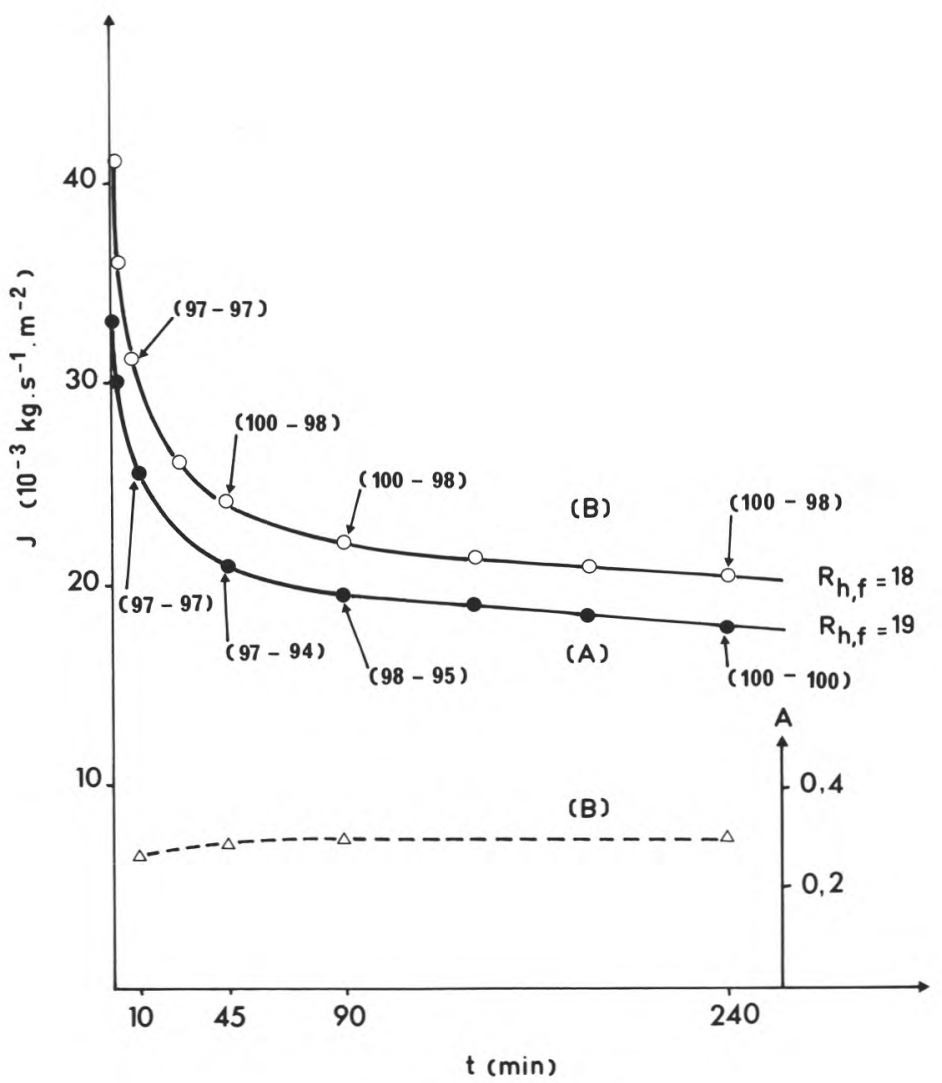

FIG. 6 a

Variation in flux $J$, rejection of $\beta$-lactoglobulin and $\alpha$-lactalbumin $(-)$, absorbance at $600 \mathrm{~nm}(A)$ and hydraulic resistance $R_{h . f}\left(10^{12} \mathrm{~m}^{-1}\right)$ versus time.

$U=4.0 \mathrm{~m} \cdot \mathrm{s}^{-1}-\Delta P=4.0 \cdot 10^{5} \mathrm{~Pa}-\theta=50^{\circ} \mathrm{C}$

$6 a(A):$ sweet whey $-p H=6.2$

(B) : centrifuged sweet whey $-\mathrm{pH}=6.2$

Evolution de la densité de flux $J$, des taux de rétention en $\beta$-lactoglobuline et $\alpha$-lactalbumine ( - ), de l'absorbance à $600 \mathrm{~nm}(A)$ et de la résistance hydraulique finale $R_{h . f}\left(10^{12} \mathrm{~m}^{-1}\right)$ en fonction du temps.

$U=4,0 \mathrm{~m} \cdot \mathrm{s}^{-1}-\Delta P=4,0 \cdot 10^{5} \mathrm{~Pa}-\theta=50^{\circ} \mathrm{C}$

$6 a(A)$ : lactosérum doux - $\mathrm{pH}=6,2$

(B) : lactosérum doux centrifugé - $p H=6,2$

$$
\left(10^{-3} \mathrm{~kg} \cdot \mathrm{s}^{-1} \cdot \mathrm{m}^{-2} \# 3.6 \mathrm{l} \cdot \mathrm{h}^{-1} \cdot \mathrm{m}^{-2}\right) .
$$


fore the increase of resistance $\Delta R_{h}$ depends on protein concentration $C_{b}, U$ and $\triangle \mathrm{P}$. SEM's observations of the membrane at the end of ultrafiltration after the final pure water flux indicate that fouling occurs at the membrane surface and within the pores at the same time (DAUFIN et al., 1987).

The relationship between protein rejection and hydraulic resistance showed that rejection of $\beta$-lactoglobulin and $\alpha$-lactalbumine were different at low $R_{h . f}$ levels (fig. 5). They got closer up to a critical $R_{h . f}$ value after which the

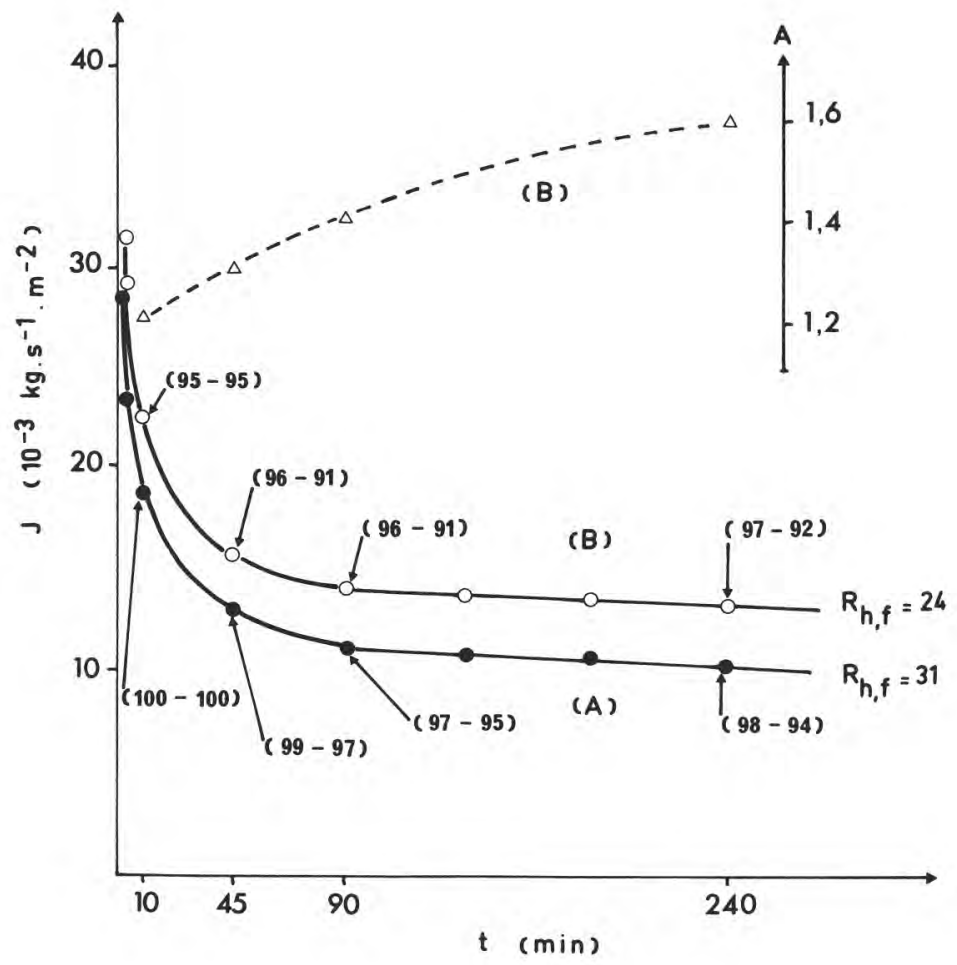

FIG. 6 b

Variation in flux $J$, rejection of $\beta$-lactoglobulin and $\alpha$-lactalbumin (-), absorbance at $600 \mathrm{~nm}(A)$ and hydraulic resistance $R_{h . f}\left(10^{12} \mathrm{~m}^{-1}\right)$ versus time.

$U=4.0 \mathrm{~m} \cdot \mathrm{s}^{-1}-\Delta P=4.0 \cdot 10^{5} \mathrm{~Pa}-\theta=50^{\circ} \mathrm{C}$

$6 b(A): R_{3}$ retentate $-p H=6.2$

(B) : centrifuged $R_{3}$ retentate - $p H=6.2$

Evolution de la densité de flux $J$, des taux de rétention en $\beta$-lactoglobuline et $\alpha$-lactalbumine ( - ), de l'absorbance à $600 \mathrm{~nm}(A)$ et de la résistance hydraulique finale $R_{h . f}\left(10^{12} \mathrm{~m}^{-1}\right)$ en fonction du temps.

$U=4,0 \mathrm{~m} \cdot \mathrm{s}^{-1}-\Delta P=4,0 \cdot 10^{5} \mathrm{~Pa}-\theta=50^{\circ} \mathrm{C}$

$6 b(A)$ : rétentat $R_{3}-p H=6,2$

(B) : rétentat $R_{3}$ centrifugé - $p H=6,2$

$$
6 b\left(10^{-3} \mathrm{~kg} \cdot \mathrm{s}^{-1} \cdot \mathrm{m}^{-2} \# 3.6 \mathrm{l} \cdot \mathrm{h}^{-1} \cdot \mathrm{m}^{-2}\right) \text {. }
$$




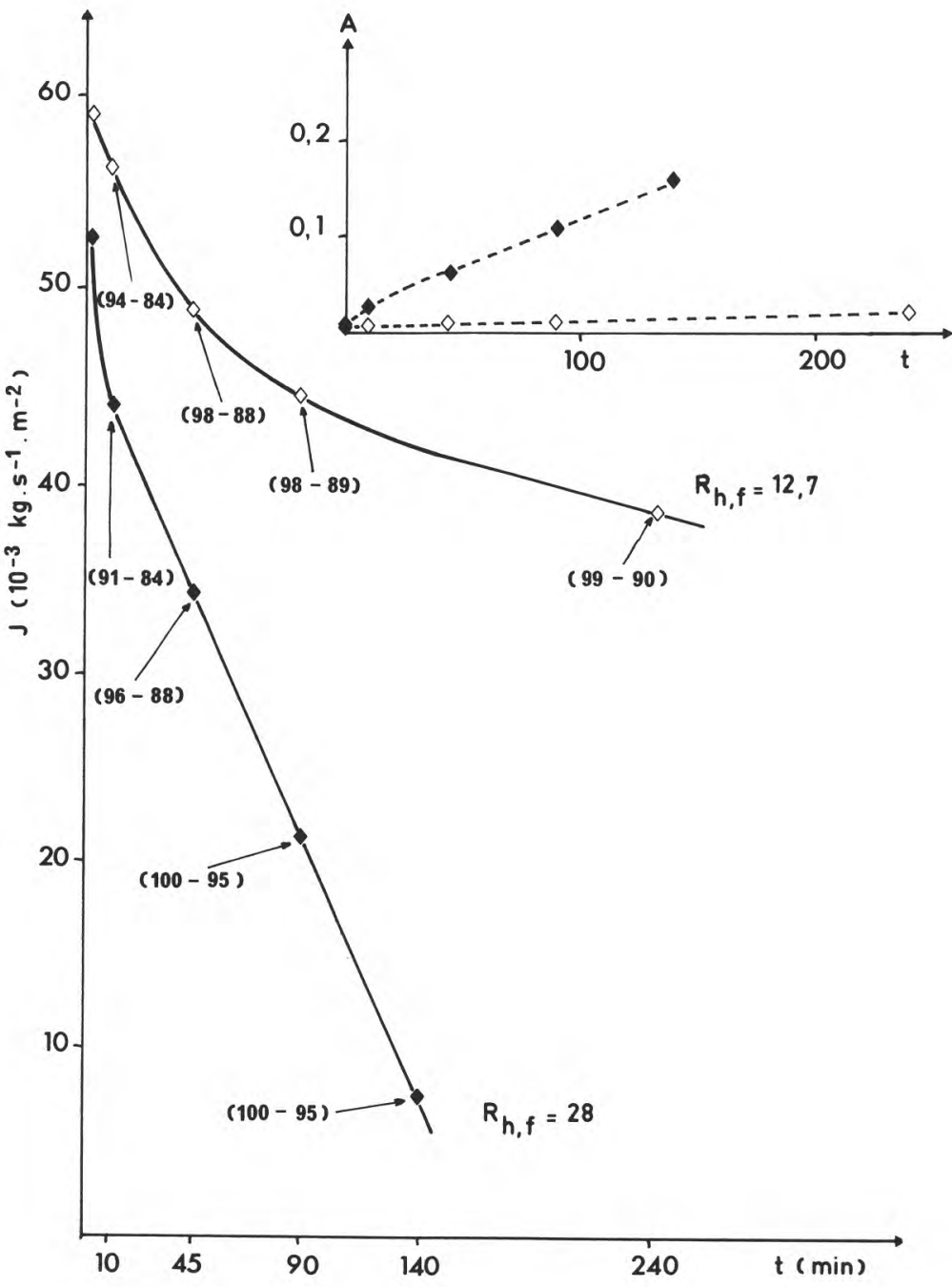

FIG. 7

Variation in flux $J$, rejection of $\beta$-lactoglobulin and $\alpha$-lactalbumin ( -$)$, absorbance at $600 \mathrm{~nm}(A)$ and final resistance $R_{h . f}\left(10^{12} \mathrm{~m}^{-1}\right)$ versus time.

$U=4.0 \mathrm{~m} \cdot \mathrm{s}^{-1}-\Delta P=4.0 \cdot 10^{5} \mathrm{~Pa}-\theta=50^{\circ} \mathrm{C}$

$(\bullet)$ : clarified whey $-p H=5.9$

$(\diamond)$ : clarified whey with citrate added $-p H=5.9$

Evolution de la densité de flux $J$, des rétentions en $\beta$-lactoglobuline et a-lactalbumine $(-)$, de l'absorbance à $600 \mathrm{~nm}$, de la résistance finale $R_{h . f}\left(10^{12} \mathrm{~m}^{-1}\right)$ en fonction $d u$ temps.

$U=4,0 \mathrm{~m} \cdot \mathrm{s}^{-1}-\Delta P=4,0 \cdot 10^{5} \mathrm{~Pa}-\theta=50^{\circ} \mathrm{C}$

( ) : lactosérum clarifié - $p H=5,9$

$(\diamond)$ : lactosérum clarifié additionné de citrates - $p H=5,9$

$\left(10^{-3} \mathrm{~kg} \cdot \mathrm{s}^{-1} \cdot \mathrm{m}^{-2} \# 3.6 \mathrm{l} \cdot \mathrm{h}^{-1} \cdot \mathrm{m}^{-2}\right)$. 
rejection of both proteins was total. This critical value $R_{h . f}$ depended on concentration : $20 \cdot 10^{12} \mathrm{~m}^{-1}$ for sweet whey and $50 \cdot 10^{12} \mathrm{~m}^{-1}$ for concentrated wheys. This would indicate that the irreversible deposit formed has a texture so as to avoid $\beta$-lactoglobulin and $\alpha$-lactalbumin to pass through the membrane.

\section{B. Influence of whey composition}

All fluids were ultrafiltrated with the same operating conditions $\left(\mathrm{U}=4.0 \mathrm{~m} \cdot \mathrm{s}^{-1} ; \Delta \mathrm{P}=4.0 \cdot 10^{5} \mathrm{~Pa}\right)$.

The shape of $\mathbf{J}=\mathrm{f}(\mathrm{t})$ curves was not affected by centrifugation prior to ultrafiltration (fig. 6). Such a pretreatment improved permeation all the more as the fluid contained suspended matter, that is whey CF was high (fig. 6b). Hydraulic resistance was lower when the suspended matter content of the fluid was low. Protein rejection slighty decreased when whey was centrifuged; this effect was greater for $R_{3}$ retentate. There was no particular change in protein rejection versus time as affected by centrifugation.

Nevertheless, the turbidity of centrifuged wheys grew during ultrafiltration indicating an increase in suspended matter content or a change in the average size of the particles (fig. 6a-6b).

For clarified whey (according to the method suggested in 1985 by FAUQUANT et al.) flux was higher than that of sweet or centrifuged whey during the first ninety minutes of ultrafiltration (fig. 7 and $6 \mathrm{a}$ ). Unfortunately, the decline of flux versus time was so steep for this kind of clarified whey ${ }^{(*)}$ that the ultrafiltration run had to be stopped after $140 \mathrm{~min}$ (fig. 8). Final $\mathbf{R}_{\mathrm{h} . \mathrm{f}}$ resistance was relatively important $\left(28 \cdot 10^{12} \mathrm{~m}^{-1}\right)$ and protein rejection increased up to $95 \%$ for $\alpha$-lactalbumin and $100 \%$ for $\beta$-lactoglobulin.

Adding citrate to clarified whey in order to complex excess of ionic calcium improved flux; $\mathrm{R}_{\mathrm{h} . \mathrm{f}}$ got lower, but also does protein rejection (fig. 7).

For the $\beta$-lactoglobulin solution, flux was almost stationary after one hour (fig. 8). The $\beta$-lactoglobulin rejection slightly increased up to $97 \%$, and the final resistance was not far from the initial value $\left(11.0 \cdot 10^{12} \mathrm{~m}^{-1}\right.$ versus $\left.7.8 \cdot 10^{12} \mathrm{~m}^{-1}\right)$.

\section{Discussion}

Whey protein concentration and change in whey composition affected fluxes, protein rejection and hydraulic resistance during ultrafiltration. Our results point out the effect of some whey components on membrane fouling and confirm the part of the limiting phenomena involved.

As $\beta$-lactoglobulin solution contained no suspended matter detectable by turbidimetry and none was formed during ultrafiltration, the fouling pheno-

$\left(^{*}\right)$ Improved methods of whey clarification have been recently proposed (MAuBois et al., 1986). 


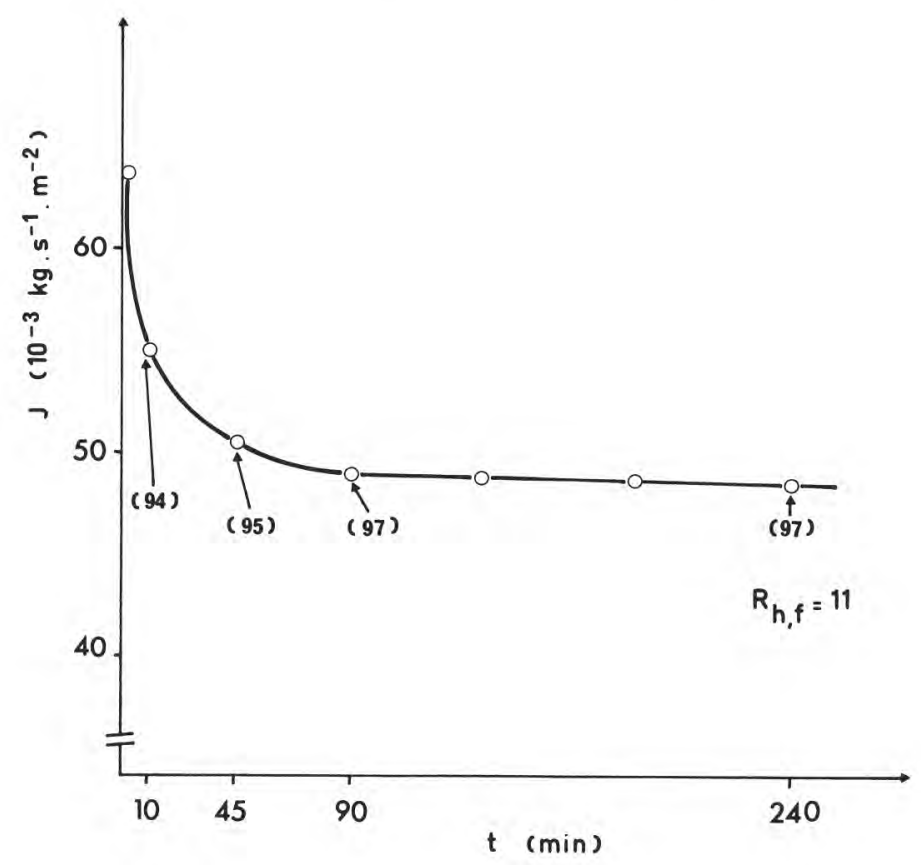

FIG. 8

Flux J, $\beta$-lactoglobulin retention ( - ) and final resistance $R_{h . f}\left(10^{12} \mathrm{~m}^{-1}\right)$ versus time during ultrafiltration of $\beta$-lactoglobulin solution at $3.0 \mathrm{~g} \cdot \mathrm{F}^{-1}(\mathrm{pH}=6.2$ - ionic strength $\left.=0.1 \mathrm{M} \cdot \mathrm{kg}^{-1}\right)$.

$U=4.0 \mathrm{~m} \cdot \mathrm{s}^{-1}-\Delta P=4.0 \cdot 10^{5} \mathrm{~Pa}-\theta=50^{\circ} \mathrm{C}$

$$
\left(10^{-3} \mathrm{~kg} \cdot \mathrm{s}^{-1} \cdot \mathrm{m}^{-2} \# 3.6 \mathrm{l} \cdot \mathrm{h}^{-1} \cdot \mathrm{m}^{-2}\right) \text {. }
$$

Densité de flux $J$, rétention de la $\beta$-lactoglobuline ( - ) et résistance hydraulique finale $R_{h . f}\left(10^{12} \mathrm{~m}^{-1}\right)$ en fonction du temps lors de l'ultrafiltration d'une solution de $\beta$-lactoglobuline à $3,0 \mathrm{~g} \cdot \mathrm{l}^{-1}\left(\mathrm{pH}=6,2\right.$ - force ionique $\left.=0,1 \mathrm{M} \cdot \mathrm{kg}^{-1}\right)$.

$U=4,0 \mathrm{~m} \cdot \mathrm{s}^{-1}-\Delta P=4,0 \cdot 10^{5} \mathrm{~Pa}-\theta=50^{\circ} \mathrm{C}$

mena involved here may be protein adsorption and/or a possible gelification of the polarization layer. Howell et al. (1981) suggested such a scheme to explain flux decline during ultrafiltration of protein solutions. MATTHIASSON (1983) showed that the adsorption equilibrium of a $2.0 \mathrm{~g} \cdot 1^{-1}$ serum-albumin solution on membrane is reached within one hour, which time would be equivalent here for $\beta$-lactoglobulin solution.

Whey clarification according to FAUQUANT et al. (1985) resulted in a residual fat removal as part of some other components such as calcium, phosphate, $\beta$-lactoglobulin and immunoglobulins. As ultrafiltration was performed at $50{ }^{\circ} \mathrm{C}$ and $\mathrm{pH}=5.9$ ( $\mathrm{pH}$ of whey after clarification), the high calcium content of clarified whey $\left(1.0 \mathrm{~g} \cdot \mathrm{1}^{-1}\right.$ versus $0.32 \mathrm{~g} \cdot 1^{-1}$ in sweet whey) may 
initiate calcium phosphate precipitation (LySTER, 1979), which is probably accelerated by the residence time of whey in our loop. This would explain increase in whey turbidity and severe decline in flux, high $\mathrm{R}_{\mathrm{h} . \mathrm{f}}$ value and increase in protein rejection (fig. 7). MERIN (1979) and LEE (1977) also suggested that membrane fouling may be due to a precipitation of calcium phosphate during whey ultrafiltration.

This assumption is experimentally confirmed by complexing calcium (fig. 7). Nevertheless, the amount of citrate added is too low to ensure a complete $\mathrm{Ca}^{2+}$ complexation and subsequently avoid calcium precipitation (revealed by a slight increase in turbidity). Furthermore, permeates were always clear and transparent showing that calcium phosphate precipitates were retained within the membrane or on the surface.

The values of fluxes, $\beta$-lactoglobulin rejection and hydraulic resistance for Ca complexed-clarified whey were close to those of $\beta$-lactoglobulin solution. The slight difference may be due to the presence of other proteins also adsorbed into the membrane and involved in the concentration polarization layer.

Centrifuging sweet whey before ultrafiltration contributed to remove the suspended matter a part of which is undoubtedly a poorly soluble more or less complex calcium salt (table 1). These particles fouled the membrane by convective deposition and therefore take part in the decline of flux at the very beginning of the ultrafiltration run. However, they did not represent the main fouling source since solvant flux and solute retention are only slightly modified. According to Kuo and CHERYAN (1983), the effect of centrifugation should be related to whey composition and specific membrane-solute interactions.

The main initial difference between clarified and sweet whey is the absence of lipoproteins in the former (FAUQuant et al., 1985). These are considered as one of the main fouling components (Maubors et al., 1986) together with residual fat (MERIN et al., 1983). Their active part in fouling phenomena satisfactorily explains the enhanced flux performance observed during at least one hour with clarified whey.

Increasing whey protein concentration contributes to high membrane fouling increase and to consequent flux decrease (fig. 1 and 2) but protein rejection is generally not very affected (fig. $3 \mathrm{c}$ ). We pointed out a relationship between hydraulic resistance $R_{\text {h.f }}$ and protein rejection. Devereux and HoARE (1986) suggested a similar relationship for the ultrafiltration of soja protein precipitates. In our experiment, a critical value of $R_{h}$ from which total protein rejection is achieved is evidenced (fig. 5). As protein retention was measured during the ultrafiltration, the observed variations in retention could also be due to the reversible phenomena which were removed by the rinsing procedure, i.e. polarization layer and a possible part of reversible fouling. Subsequently, since $R_{h}$ is accounting for $\Delta R_{h}$, irrv, that is the so-called irreversible fraction of the fouling, some caution must be brought when reading and interprating the relation rejection versus final hydraulic resistance. The critical value of $R_{h}$ was about twice greater for concentrated wheys than for sweet whey. This indicates that soluble proteins take part in membrane fouling, but other components also are involved since the critical value of $R_{h . f}$ was the 
same for $R_{3}$ and $R_{8}$ concentrates. Indeed, the preparation of Whey Protein Concentrate (WPC) by ultrafiltration also results in concentrating suspended matter (such as microorganisms, inorganic precipitates or protein aggregates), which cannot pass through the membrane.

For whey concentrates, changes in operating conditions are less likely to enhance fluxes because of the high ability of these concentrates to foul the membrane. Moreover, final values of membrane hydraulic resistance and protein rejection decrease with increasing velocity $(\mathrm{U})$ or decreasing pressure $(\Delta \mathrm{P})$. Hence, the relationship between $\mathrm{R}_{\mathrm{h}, \mathrm{f}}$ and rejection seems to be due to a secondary membrane building-up (protein and insoluble particles) but also to a true « foulant " inside the pores since the final value of $R_{h, f}$ are often higher.

The effects of modifications in whey composition on flux and protein rejection give an insight into the phenomena involved in membrane fouling : protein adsorption and convective deposition of suspended matter. Both phenomena occur at the very beginning of an ultrafiltration run. Their extent and kinetics are related to whey composition and operating conditions (fig. 2). Protein rejection also is affected by whey composition and operating conditions and therefore membrane selectivity depends on fouling type as noticed by HidDink et al. (1981) and NAKAO and Kimura (1981). However, mass transfer is also related to concentration polarization : a polarization layer building-up occurs within the first minute according to CHUDACEK and FANE (1984) as already reported for sweet whey ultrafiltration (TADDEI et al., 1986).

Despite a lack of quantitative analysis of these limiting phenomena, we may bring out some trends towards improving the productivity of ultrafiltration plants. These recommendations must be fitted to the combination membranesolution studied since mass transfer depends on such a combination. On a qualitative point of view, it is advisable to start ultrafiltration at a low flowvelocity in order to enhance protein rejection since the membrane is poorly retentive. Afterwards, an increase in flow-velocity is required to prevent higher fouling: protein content of the permeate must be checked continuously to maintain a high rejection capacity by controlling pressure.

However, reducing membrane fouling improves flux value but decreases protein rejection and vice-versa. Hence, it appears an opposition between high flux and high rejection with partly retentive membranes, which in fact requires to come to a compromise. For example, on the basis of our results, we could define operating conditions $(\mathrm{U}, \Delta \mathrm{P})$ permitting a rejection (98\% at least) for both proteins, together with the highest fluxes. In a three-stage ultrafiltration plant, operating conditions should be adapted to each of them. The first one should work with sweet whey at $4.0 \mathrm{~m} \cdot \mathrm{s}^{-1}-5.0 \cdot 10^{5} \mathrm{~Pa}$ and the third with $\mathrm{R}_{8}$ retentate at $3.0 \mathrm{~m} \cdot \mathrm{s}^{-1}-6.0 \cdot 10^{5} \mathrm{~Pa}$. For the second stage, $\mathrm{R}_{3}$ retentate should be processed with intermediate operating conditions. Moreover, the value of the final flux after a $4 \mathrm{hr}$-run would then be $19.1 \cdot 10^{3} \mathrm{~kg} \cdot \mathrm{s}^{-1} \cdot \mathrm{m}^{-2}$ $\left(68,81 \cdot \mathrm{h}^{-1} \cdot \mathrm{m}^{-2}\right)$ for sweet whey and only $10.1(36.4)$ for $R_{8}$ retentate, because of the high $R_{h . f}$ observed. From this work it appears that $R_{h, f}$ is an important characteristic of UF system since fluxes and rejection are depending on it tightly. It is related to operating conditions $(\mathrm{U}, \Delta \mathrm{P})$ and concentration factor. It could be of interest to find out an equation type as :

$$
R_{h}=A(C F)^{\alpha} U^{\beta}(\Delta P)^{\gamma}
$$


Membrane fouling must also be taken into account considering a possible difficulty in membrane cleaning as related with high $R_{\text {h.f }}$. We had to cope with this problem for clarified whey and $R_{8}$ retentate and the previously used cleaning procedure (TADDEI et al., 1986) had to be modified (cf. Experimental part).

Or course, other compromises may be found to answer to the user's requirements : improvement of the WPC's protein content (no losses of proteins) or quantity (a maximum whey volume treated per day).

\section{Conclusion}

This work emphazises the effect of whey composition on fouling and limiting flux during ultrafiltration.

Several whey components are involved in membrane fouling: soluble proteins, suspended particles, residual fat and calcium able to precipitate as phosphate during ultrafiltration. They are involved in two kinds of phenomena : adsorption and convective deposition occuring at the very beginning of the run. Higher whey protein concentrations increase the extent of these limiting phenomena.

It also appears clearly that membrane selectively is not an intrinsic value but depends on fouling type. which is closely related to solution composition and operating conditions.

From our results, an optimization of operating conditions during whey concentration by ultrafiltration was proposed. However, a compromise between quality and quantity for whey protein concentrates must be defined because of the opposition between high flux and high protein rejection for partly retentive membranes.

Reçu le 5 février 1987. Accepté pour publication le 2 juin 1987.

\section{Acknowledgements}

We thank Mr. J.L. MAUBoIS and $\operatorname{Pr}$ G. BRULÉ for their helpful advices all along the work. Our colleague's technical assistance, Mr. M. RoIGNANT (real time data aquisition), Mr. J. SAMSON (retentates preparation) and Mrs. F. MICHEL (some experiments and analysis) has been highly appreciated. Mr. J.P. LABBE's (ENSC Paris) and Mrs. A. BourRochE's (Unité Centrale de Documentation, INRA, Jouy-en-Josas, France) contribution for improving the translation of this paper is gratefully acknowledged. 


\section{References}

Baklouti S., Aimar P., Sanchez V., 1984. Adsorption et transfert de matière en ultrafiltration. Lait, 64, 206-216.

Blatt W.F., David A., Michaels A.S., Nelson L., 1970. Solute polarization and cake formation in membrane ultrafiltration: causes, consequences and control techniques. In: Membrane Science and Technology, Flinn J.E. Ed., Plenum Press, New York, 47-97.

Cheryan M., Kuo K.P., 1984. Hollow fibers and spiral wound modules for ultrafiltration of whey: energy consumption and performance. J. Dairy Sci., 67, 1407-1413.

Chudacek M.W., Fane A.G., 1984. The dynamics of polarization in unstirred and stirred ultrafiltration. J. Membrane Sci., 21, 145-160.

Daufin G., Michel F., Quemerais A., 1987. Work in progress.

DE Boer R., Hiddink J., 1980. Membrane processes in the dairy industry: state of the art. Desalination, 35, 169-192.

Devereux N., Hoare M., 1986. Membrane separation of protein precipitates : studies with crossflow filtration. Biotechnol. Bioeng., 28, 422-431.

Fauquant J., Vieco S., Brulé G., Maubois J.L., 1985. Clarification des lactosérums doux par agrégation thermocalcique de la matière grasse résiduelle. Lait, 65, 1-20.

Forsum E., 1977. Use of a whey protein concentrate as a supplement to maize, rice and potatoes. A chemical and biological evaluation using growing rate. J. Nutr., 105, 147-153.

Hayes J.F., Dunkerley J.A., Muller L.L., Griffin A.T., 1974. Studies on whey processing by ultrafiltration. II. Improving permeation rates by preventing fouling. Aust. J. Dairy Technol., 29, $132-140$.

Hiddink J., DE Boer R., Nooy P.F.C., 1981. Effect of various pretreatments on the ultrafiltration of sweet cheese whey at about $55^{\circ} \mathrm{C}$. Milchwissenschaft, $36(11), 657-663$.

HIDDINK J., VAN DER WAAL M.J., 1984. Evaluation of hyperfiltration systems for sweet cheese whey . I. Experimental results with a single pass and a recirculation system. J. Food Eng., 3, 225-239,

Howell J.A., Velicangil O., Le M.S., Herrera A.L., 1981. Ultrafiltration of protein solutions. Am. N.Y, Acad. Sci., 369, 355-366.

Kosinsky A.A., LightFoot E.N., 1972. Proteins ultrafiltration, a general example of boundary layer filtration. AIChE J., 18 (5), 1030-1041.

Kuo K.P., Cheryan M., 1983. Ultrafiltration of acid whey in a spiral wound unit. Effect of operating parameters on membrane fouling. J. Food Sci., 48 (4), 1113-1117.

LeE D.N., Merson R.L., 1976a. Chemical treatments of cottage cheese whey to reduce fouling of ultrafiltration membranes. J. Food Sci., 58 (10), 1423-1432.

Lee D.N., Merson R.L., 1976b. Prefiltration of cottage cheese whey to reduce fouling of ultrafiltration membranes. J. Food Sci., 41, 403-410.

LEE R.C., 1977. Serum factors affecting permeation and fouling of selected UF membranes. Ph. D. Thesis, Ohio State University, Ohio (USA).

LYSTER R.L.J., 1979. The equilibrium of calcium and phosphate ion with the micellar calcium phosphate in cow's milk. J. Dairy Res., 46, 343-346.

Matthiasson E., 1983. The role of macromolecular adsorption in fouling of ultrafiltration membranes. J. Membrane Sci., 16, 23-36.

Maubois J.L., 1980. Ultrafiltration of whey. J. Soc. Dairy Technol, 33 (2), 55-58.

Maubois J.L., Pierre A., Fauquant J., Piot M., 1986. Industrial fractionation of main whey proteins. Int. Whey Conf., Chicago, USA.

Melachouris N., 1984. Critical aspects in development of whey protein concentrate. J. Dairy Sci., $67,2693-2700$.

MERIN U., 1979. A study of the mechanism of fouling of ultrafiltration membranes. Ph. D. Thesis, University of Illinois, Urbana Champaign, Illinois (USA). 
Merin U., Gordin S., Tanny G.B., 1983. Microfiltration of sweet cheese whey. N.Z. J. Dairy Sci. Technol., 18, 153-160.

Muller L.L., Hayes J.F., 1973. Studies on whey processing by ultrafiltration. Comparison of various ultrafiltration modules on whey from hydrochloric acid whey and cheddar cheese. Aust. J. Dairy Technol., 28 (2), 70-77.

Nakao S.I., Kimura S., 1981. Analysis of solute rejection in ultrafiltration. J. Chem. Eng. Jap., 14, 32-37.

Pierre A., Fauquant J., 1986. Principes pour un procédé industriel de fractionnement des protéines du lactosérum. Lait, 66 (4), 405-420.

TADDEI C., 1986. Mécanismes influençant le transfert de matière lors de l'ultrafiltration de lactosérum doux. Thèse $\mathrm{D}^{\text {r}}$-Ing., UPS, Toulouse, France.

Taddei C., Aimar P., Daufin G., Sanchez V., 1986. Etude du transfert de matière lors de l'ultrafiltration de lactosérum doux sur membrane minérale. Lait, 66 (4), 371-380.

ZALL R.R., 1982. WPC update : product processing and properties Proc. Whey Products Conference, Schaumburg, Illinois (USA). 